\title{
On Conformal Symmetric Tensor of Kaehlerian Manifolds
}

\section{U.S. Negi ${ }^{1} \cdot$ Sulochana $^{1}$}

${ }^{1}$ Department of Mathematics, H.N.B. Garhwal University (A Central University), S.R.T. Campus Badshahithaul249199, Tehri Garhwal, Uttarakhand.

*Corresponding author: usnegi7@gmail.com

Received: 27.6.2021; Revised: 10.11.2021; Accepted:12.11.2021

CSociety for Himalayan Action Research and Development

Abstract: Chaki (1956) has obtained some theorems on recurrent and ricci-recurrent spaces. After that, Negi (2017) has calculated theorems on almost product and decomposable spaces. Also, Negi et al. (2019) has studied an analytic HP-transformation in almost Kaehlerian spaces. In this paper, we have defined and considered on symmetric tensor and conformal symmetric tensor of Kaehlerian manifolds and some theorems established.

Key Words: Kaehlerian manifolds, conformal symmetric tensor and Ricci-recurrent spaces.

MSC 2010: 53C15, 53C55, 53B35.

\section{Introduction:}

An $\boldsymbol{n}$-dimensional Kaehlerian manifolds $K_{n}(n \geq 2)$ will be called conformal symmetric tensor if the conformal curvature tensor will be:

$$
\begin{aligned}
C^{h}{ }_{i j k}=R^{h}{ }_{i j k}+\frac{1}{n-2}\left(\delta^{h}{ }_{j} R_{i k}-\delta^{h}{ }_{k} R_{i j}\right. & \left.+g_{i k} R^{h}{ }_{j}-g_{i j} R^{h}{ }_{k}\right) \\
& +\frac{R}{(n-1)(n-2)}\left(\delta^{h}{ }_{k} g_{i j}-\delta^{h}{ }_{j} g_{i k}\right) \\
C^{h}{ }_{i j k, l} & =0
\end{aligned}
$$

where (, ) denote covariant differentiation with respect to the metric tensor $g_{i j}$ of $K_{n}$. By easy calculation, we have

$$
C_{i j k l} C^{i j k l}=R_{i j k l} R^{i j k l}-\frac{4 R_{i j} R^{i j}}{(n-2)}+\frac{2 R^{2}}{(n-1)(n-2)}
$$

used for $K_{n \text {, then }}\left(C_{i j k l} C^{i j k l}\right)_{, m}=0$. Hence $C_{i j k l} C^{i j k l}$ is constant.

While the scalar $\quad C_{i j k l} C^{i j k l}$ is constant for $K_{n}$, the scalars $\quad R_{i j k l} R^{i j k l}$ and $R_{i j} R^{i j}$ are not necessarily consequently. Thus, form (1.3) it follows that the scalar curvature of $K_{n}$ is of constant scalar curvature iff the first covariant derivative of the Ricci tensor is a symmetric tensor. From (1.2) we obtain [Chaki (1956)]:

$$
C^{h}{ }_{i j k, h}=\frac{n-3}{n-2}\left[R_{i j, k}-R_{i k_{j} j}+\frac{1}{2(n-1)}\left(g_{i k} R_{j j}-g_{i j} R_{, k}\right)\right]=0
$$


from (1.4) and then knowing $(n>3)$, we get:

$$
R_{i j, k}-R_{i k, j}=\frac{1}{2(n-1)}\left(g_{i j} R_{, k}-g_{i k} R_{j}\right)
$$

$$
\begin{aligned}
& \text { If } \quad R_{\text {is constant, } \quad-\quad R_{i j, k}}-R_{i k_{j} j}=0 . \text { That is, the tensor } \\
& R_{i j, k} \text { is a symmetric tensor. Equally, if } \quad R_{i j, k} \text { be a symmetric tensor, from (1.5) }
\end{aligned}
$$

we get:

$g_{i j} R_{, k}=g_{i k} R_{j j}$

whence $R_{, k}=0$, so $\mathrm{R}$ is constant.

Again, $\quad K_{n}$ of constant scalar curvature $\quad R_{i j, k} \quad-\quad R_{i k, j}=0$.

Other than for such a recurrent space $\quad R_{i j, k l} \quad-\quad R_{i j, l k}$, which is not, in general zero, can be expressed in terms of the metric tensor and the second covariant derivatives of the tensor $\quad R_{j}^{i}$.

The circumstances of integrability of the equations (1.2) are:

$C^{h}{ }_{\alpha j k} R^{\alpha}{ }_{i l m}+C^{h}{ }_{i \alpha k} R^{\alpha}{ }_{j l m}+C^{h}{ }_{i j \alpha} R^{\alpha}{ }_{k l m}-C^{\alpha}{ }_{i j k} R^{h}{ }_{\alpha l m}=0$

We can state (1.6) when:

$$
\begin{aligned}
&\left(R^{h}{ }_{i j k, l m}-R^{h}{ }_{i j k, m l}\right)+ \frac{1}{n-2}\left[\delta^{h}{ }_{j}\left(R_{i k, l m}-R_{i k, m l}\right)-\delta^{h}{ }_{k}\left(R_{i j, l m}-R_{i j, m l}\right)\right. \\
&+g_{i k}\left(R^{h}{ }_{j, l m}-R^{h}{ }_{j, m l}\right) \\
&\left.-g_{i j}\left(R^{h}{ }_{k, h m}-R^{h}{ }_{k, m h}\right)\right]=0
\end{aligned}
$$

Contracting $h$ and $l$ we get:

$$
\begin{aligned}
& \left(R^{h}{ }_{i j k, h m}-R^{h}{ }_{i j k, m h}\right)+\frac{1}{n-2}\left[\left(R_{i k j m}-R_{i j, k m}\right)+\left(R_{i j, m k}-R_{i k, m j}\right)\right. \\
& +g_{i k}\left(R_{j, h m}^{h}-R_{j, m h}^{h}\right)-g_{i j}\left(R_{k, h m}^{h}-R_{k, m h}^{h}\right)=0
\end{aligned}
$$

If a recurrent space is of constant scalar curvature then $R_{i j, k}=R_{i k, j}$ therefore,

$R^{h}{ }_{i j k, h}=0$

Hence (1.8) reduces to:

$R_{i j k, m h}^{h}=\frac{1}{n-2}\left[\left(R_{i m, j k}-R_{i m, k j}\right)+\left(g_{i j} R_{k, m h}^{h}-g_{i k} R_{j, m h}^{h}\right)\right]$

Again in a Kaehlerian manifolds $K_{n}$, then:

$R^{h}{ }_{i j k, h m}-R^{h}{ }_{i j k, m h}+R^{h}{ }_{m j k, h i}-R^{h}{ }_{m j k, i h}-R_{i m, j k}+R_{i m, k j}=0$

Hence in asset of (1.9), we get: 
$R_{i m, k j}-R_{i m, j k}=R^{h}{ }_{i j k, m h}+R_{m j k, i h .}^{h}$.

Therefore from (1.10), then:

$R_{i m, j k}-R_{i m, k j}=\frac{1}{n}\left(g_{i k} R_{j, m h}^{h}+g_{m k} R_{j, i h}^{h}-g_{i j} R_{k, m h}^{h}-g_{m j} R_{k, i h)}^{h}\right.$

That is $R_{i m, j k}-R_{i m, k j}$ for $K_{n}$ of constant scalar curvature in terms of the metric tensor $g_{i j}$ and the second covariant derivatives of the tensor $R_{j}^{h}$.

\section{On Symmetric Tensor of Kaehlerian Manifolds:}

Theorem (2.1): Every Einstein symmetric tensor of Kaehlerian manifolds $K_{n}$ is symmetric in the logic of cartan the scalar curvature of which is:

$R^{2}=\frac{n(n-1)}{2}\left[R_{i j k l} R^{i j k l}-C_{i j k l} C^{i j k l}\right]$.

Proof: Let $K_{n}$ be an Einstein manifold. Then we have:

$R_{i j}=\frac{R}{n} g_{i j}$.

since $(n>3), R$ is constant. Therefore $R_{i j, p}=0$.

Hence from (1.2),

$R^{h}{ }_{i j k, l}=0$.

Since $\mathrm{R}$ is constant and the manifold is an Einstein manifold $R_{i j} R^{i j}$ is also constant. Hence from (1.3) it follows that $R_{i j k l} R^{i j k l}$ is also a constant. This is also obvious from (2.1).

Since $R_{i j} R^{i j}=\frac{R^{2}}{n}$, we have from (1.3), then:

$C_{i j k l} C^{i j k l}=R_{i j k l} R^{i j k l}-\frac{2 R^{2}}{n(n-1)}$,

where $\left.R^{2}=\frac{n(n-1)}{2}{ }_{[} R_{i j k l} R^{i j k l}-C_{i j k l} C^{i j k l}\right]$

Hence we get the result.

Theorem (2.2): Every recurrent Kaehlerian manifolds $K_{n}$ is conformal smooth and Riccirecurrent provided that $R_{i j k l, a}+\lambda_{a}\left(C_{i j k l}-R_{i j k l}\right)=0$, where $\lambda_{a}$ is a non-zero vector.

Proof: Let $K_{n}$ is a recurrent space individual by a non-zero vector $\lambda_{a}$. Then [Brinkmann (1924)],

$C_{i j k l m}=\lambda_{m} C_{i j k l}$. 
Since $C_{i j k l, m}=0, \quad \lambda_{m} C_{i j k l}=0$

whence $C_{i j k l=0}$.

After that, we assume that $K_{n}$ is a Ricci- recurrent space individual by a non-zero vector $\lambda_{a}$, therefore:

$$
\begin{aligned}
C_{i j k l, a}=R_{i j k l, a}+ & \frac{1}{n-2}\left(g_{i k} R_{j l, a}-g_{i l} R_{j k, a}+g_{j l} R_{i k, a}-g_{j k} R_{i l, a}\right) \\
& +\frac{R_{a}}{(n-1)(n-2)}\left(g_{i l} g_{j k}-g_{i k} g_{j l}\right),
\end{aligned}
$$

We have in this case:

$$
R_{i j k l, a}+\lambda_{a}\left(C_{i j k l}-R_{i j k l}\right)=0
$$

Conversely, if (2.3) holds:

$R_{i l, a+} \lambda_{a}\left(C_{i l}-R_{i l)}=0, \quad\right.$ where $R_{i l, a}=\lambda_{a} R_{i l}$. Hence proved.

Theorem (2.3): If Kaehlerian manifolds $K_{n}$ be Ricci-recurrent then its vector of recurrent is a null vector and the rank of the matrix $\left(R_{i j}\right)$ is one.

Proof: We now assume that $K_{n}$ is a Ricci-recurrent space, the vector of recurrences of which is a gradient. Then, [Rahman (1970)],

$R_{i j, k l}-R_{i j, l k}=0$

Again, for a non-decomposable Ricci-recurrent space, $R=0$.

Hence, using formula (1.10), we have

$g_{i k} R^{h}{ }_{j, m h}+g_{m k} R_{j, i h}^{h}-g_{i j} R_{k, m h}^{h}-g_{m j} R_{k, i h}^{h}=0$

Thus, if Kaehlerian manifolds $K_{n}$ be Ricci-recurrent with its vector of recurrence as a gradient, then (2.4) holds. From non-decomposable Ricci-recurrent $K_{n}$, that is:

$R_{i j, k}=R_{i k, j} \Rightarrow \lambda_{k} R_{i j}=\lambda_{j} R_{i k}$, then:

$R_{i j}=\mathrm{r} \lambda_{i} \lambda_{j} \quad(\mathrm{r} \neq 0)$

where $\mathbf{r}$ is a scalar factor of proportionality.

from (2.5), we get: $\quad g^{i j} R_{i j}=\mathrm{r} g^{i j} \lambda_{i} \lambda_{j}$.

where $\quad g^{i j} \lambda_{i} \lambda_{j}=0$.

Hence proved.

Theorem (2.4): Every projective-symmetric Kaehlerian manifolds $K_{n}$ is symmetric tensor in the logic of cartan. 
Proof: Since every Cartan-symmetric space is both projective-symmetric manifold and conformally symmetric manifold, it follows from the theorem 2.1 that Cartan-symmetric manifold are the only manifold which are both projective-symmetric manifold and conformally symmetric manifold [Canfes (2006)],

We now consider a Kaehlerian manifolds $K_{n}$ in which the weyl projective curvature tensor $W^{h}{ }_{i j k}=R^{h}{ }_{i j k}-\frac{1}{n-1}\left(\delta^{h}{ }_{k} R_{i j}-\delta^{h}{ }_{j} R_{i k}\right)$

and

$W^{h}{ }_{i j k, l}=0$

An n-dimensional Kaehlerian manifolds $K_{n}$ satisfying (2.7) has been called Projective-symmetric manifold. We know that for such a space, $R_{i j, k}$ is a symmetric tensor in case $(n \neq 2)$. In asset of (2.7), it follows from (2.1) and (2.6) that:

$$
\begin{aligned}
R^{h}{ }_{i j k, l} & =-\frac{1}{n-2}\left(\delta^{h}{ }_{j} R_{i k, l}-\delta^{h}{ }_{k} R_{i j, l}+g_{i k} R_{j, l}^{h}-g_{i j} R_{k, l}^{h}\right) \\
& =\frac{1}{n-1}\left(\delta^{h}{ }_{k} R_{i j, l}-\delta^{h}{ }_{j} R_{i k, l}\right) .
\end{aligned}
$$

Hence, we get:

$g_{i k} R^{h}{ }_{j, l}-g_{i j} R_{k, l}^{h}=\frac{1}{n-1}\left(\delta^{h}{ }_{k} R_{i j, l}-\delta^{h}{ }_{j} R_{i k, l}\right)$,

or

$g_{i k} R_{t j, l}-g_{i j} R_{t k, l}=\frac{1}{n-1}\left(g_{t k} R_{i j, l}-g_{t j} R_{i k, l}\right)$

Therefore $R_{t i j k, l}=0$.

Thus, symmetric tensor condition proved.

\section{On Conformal Symmetric Tensor of Kaehlerian Manifolds:}

Theorem (3.1): Given in terms of the conformal symmetric tensor, that the conformal map of a Kaehlerian Manifolds $K_{n}$ be a recurrent space specified by a non- zero vector $\lambda_{p}$ is that following:

$$
\begin{aligned}
\lambda_{l} C_{h i j k}-{ }_{2} C_{h i j k} \sigma_{l}=-[ & C_{l i j k} \sigma_{h}+C_{h l j k} \sigma_{i}+C_{h i l k} \sigma_{j}+{ }_{h i j l} \sigma_{k} \\
& \left.-\sigma^{s}\left(g_{h l} C_{s i j k}+g_{i l} C_{h s j k}+g_{j l} C_{h i s k}+g_{k l} C_{h i j s}\right)\right]
\end{aligned}
$$

Proof: Let $K_{n}{ }^{*}(\mathrm{n}>3)$ be a space with the metric tensor:

$g^{*}{ }_{i j}=e^{2 \sigma} g_{i j}$ 
Where $g_{i j}$ is the metric tensor of a $K_{n}$.

Then $K_{n}{ }^{*}$ is said to be a conformal map of $K_{n}$. A conformal map shall be called proper if it arises from a function $\sigma$ for which $\Delta_{1} \sigma \neq 0$. If $C^{*}{ }_{i j k}$ and $C^{* t}{ }_{i j k}$ be Weyl's conformal curvature tensors for $K_{n}$ and $K_{n}{ }^{*}$, then it is known that [Walker (1950)]:

$C^{* t}{ }_{i j k}=C^{t}{ }_{i j k}$

Hence

$C^{*}{ }_{h i j k}=e^{2 \sigma} C_{h i j k}$

Let a semi - colon denote covariant differentiation with respect to $g^{*}{ }_{i j}$. Writing $\sigma_{i}=\frac{\partial \sigma}{\partial x^{i}}$ and $\sigma^{j}=g^{i j} \sigma_{i}$

We obtain from (3.3), then:

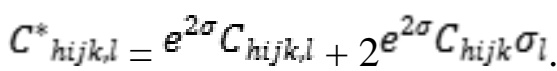

But

$$
\begin{aligned}
C_{h i j k, l=C_{h i j k, l}-4} C_{h i j k} \sigma_{l}-\left(C_{l i j k} \sigma_{h}\right. & \left.+C_{h l j k} \sigma_{i}+C_{h i l k} \sigma_{j}+C_{h i j l} \sigma_{k}\right) . \\
& +\sigma^{s}\left(g_{h l} C_{s i j k}+g_{i l} C_{h s j k}+g_{j l} C_{h i s k}+g_{k l} C_{h i j s}\right) .
\end{aligned}
$$

Therefore

$$
\begin{aligned}
C_{h i j k, l}^{*}= & e^{2 \sigma} C_{h i j k, l}-4^{2 \sigma} C_{h i j k} \sigma_{l}-e^{2 \sigma}\left[C_{l i j k} \sigma_{h}+C_{h l j k} \sigma_{i}+C_{h i l k} \sigma_{j}\right. \\
& \left.+{ }_{h i j l} \sigma_{k}-\sigma^{s}\left(g_{h l} C_{s i j k}+g_{i l} C_{h s j k}+g_{j l} C_{h i s k}+g_{k l} C_{h i j s}\right)\right]+2^{2 \sigma} C_{h i j k} \sigma_{l} \\
= & e^{2 \sigma} C_{h i j k, l}-2^{2 \sigma} C_{h i j k} \sigma_{l}-e^{2 \sigma} C_{l i j k} \sigma_{h}+C_{h l j k} \sigma_{i}+C_{h i l k} \sigma_{j} \\
& +C_{h i j l} \sigma_{k-} \sigma^{s}\left(g_{h l} C_{s i j k}+g_{i l} C_{h s j k}+g_{j l} C_{h i s k}+g_{k l} C_{h i j s)}\right)
\end{aligned}
$$

If $K_{n}$ be a recurrent space which is not conformally flat, then we get:

$$
C_{h i j k, l}=0 \quad \text { but } \quad C_{h i j k} \neq 0 .
$$

equation (3.4) can be written as:

$$
\begin{gathered}
C_{h i j k, l=-}^{*} e^{2 \sigma} C_{h i j k} \sigma_{l}-e^{2 \sigma}{ }_{[} C_{l i j k} \sigma_{h}+C_{h l j k} \sigma_{i}+C_{h i l k} \sigma_{j}+C_{h i j l} \sigma_{k} \\
\left.-\sigma^{s}\left(g_{h l} C_{s i j k}+g_{i l} C_{h s j k}+g_{j l} C_{h i s k}+g_{k l} C_{h i j s}\right)\right]
\end{gathered}
$$

If $K_{n}{ }^{*}$ be a recurrent space specified by a non- zero vector $\lambda_{p}$, then

$C^{*}{ }_{h i j k, l}=\lambda_{l} C^{*}{ }_{h i j k}=\lambda_{l} e^{2 \sigma} C_{h i j k}$.

equation (3.5) can be written as: 


$$
\begin{aligned}
\lambda_{l} C_{h i j k}-{ }_{2} C_{h i j k} \sigma_{l}=-\left[{ }_{l i j k} \sigma_{h}+\right. & C_{h l j k} \sigma_{i}+C_{h i l k} \sigma_{j}+C_{h i j l} \sigma_{k} \\
& \left.-\sigma^{s}\left(g_{h l} C_{s i j k}+g_{i l} C_{h s j k}+g_{j l} C_{h i s k}+g_{k l} C_{h i j s}\right)\right]
\end{aligned}
$$

Thus, we get the result.

Theorem (3.2): A condition the conformal map of a Kaehlerian Manifolds $K_{n}(\mathrm{n}>3)$ may be a recurrent space is that there exists a function $\sigma$ is following:

$\sigma_{s} C^{s}{ }_{i j k}+\frac{1}{n-2} R_{i j k}=0$.

Proof: If, the conformal map of a Kaehlerian Manifolds $K_{n}$ be a recurrent space then the condition reduces to (3.1) with the left-hand side equal to zero. With mentions to the equation (3.3), we have the following known equations:

$$
\begin{aligned}
e^{-2 \sigma_{R^{*}}{ }_{h i j k}=} & \left.R_{h i j k}+g_{h k}\left(\sigma_{i, j}-\sigma_{i} \sigma_{j}\right)+g_{i j(} \sigma_{h, k}-\sigma_{h} \sigma_{k}\right) \\
& -g_{h j(}\left(\sigma_{i, k}-\sigma_{i} \sigma_{k}\right)-g_{i k}\left(\sigma_{h, j}-\sigma_{h} \sigma_{j}\right)+\left(g_{h k} g_{i j}-g_{h j} g_{i k} \Delta_{l} \sigma,\right.
\end{aligned}
$$

where

$$
\sigma_{i, j}-\sigma_{i} \sigma_{j}=\frac{1}{n-2}\left(R^{*}{ }_{i j}-R_{i j)}-\frac{1}{2(n-1)(n-1)}\left(g^{*}{ }_{i j} R^{*}-g_{i j} \mathrm{R}\right)-\frac{1}{2} g_{i j} \Delta_{1} \sigma\right.
$$

The conditions of integrability of the equation (3.7) were obtained [Ficken (1939)]:

$\sigma_{s} C^{s}{ }_{i j k}=\frac{1}{n-2}\left(R^{*}{ }_{i j k}-R_{i j k}\right)$

where

$R_{i j k}=R_{i j, k}-R_{i k_{j} j}-\frac{1}{2(n-1)}\left(g_{i j} R_{, k}-g_{i k} R_{, j}\right)$

and

$R^{*}{ }_{i j k}=R^{*}{ }_{i j, k}-R^{*}{ }_{i k j}-\frac{1}{2(n-1)}\left(g^{*}{ }_{i j} R^{*}, k-g^{*}{ }_{i k} R^{*}{ }_{j}\right)$

If we now suppose that $K_{n}{ }^{*}$ is a recurrent space, than $R^{*}{ }_{i j k}=0$.

Putting $R^{*}{ }_{i j k}=0$ in (3.8) we get:

$\sigma_{s} C^{s}{ }_{i j k}+\frac{1}{n-2} R_{i j k}=0$.

Hence we get the result.

\section{References:}

Mathematische Annalen, Vol.91,

Brinkmann H.W. (1924). Riemannian pp.269-278. spaces conformal to Einstein spaces. 
Conjecture. Differential

Geometry and Dynamical Systems, 8, 34-42.

Chaki M C (1956). Some theorems on Recurrent and Ricci-recurrent spaces. Rend. Cont. del. Sem. Math. Della Univ. di Padova, Vol, pp.168-176.

Ficken F A (1939). The Riemannian and Affine differential geometry of product spaces. Annals of Mathematics (2), Vol.40, pp.892913.

Negi U S (2017). Theorems on Almost product and decomposable spaces, Aryabhatta J. Math. Inform. 9 (1), 105-110.

Negi U S, Trishna Devi, Poonia M S (2019). An analytic HPtransformation in almost Kaehlerian spaces. Aryabhatta Journal of Mathematics \& informatics, Vol. 11, No. 1, pp. 103-108,

Rahman M S (1970). Some results on second order Ricci-recurrent spaces, J. Nat. Sci. Math.10 (1), 65-69. Walker A G (1950). On Ruse's spaces of recurrent curvature, Proc. Lond. Math. Soc. 52 (2), 36-64. 